\title{
Suficiência amostral para a estimação da produção de cana-de-açúcar
}

\author{
Sample sufficiency for sugarcane production estimating
}

\author{
Jefferson Gonçalves Acunha ${ }^{\mathrm{I}}$ Ricardo Augusto de Oliveira' ${ }^{\mathrm{II}}$ Walmes Marques Zeviani ${ }^{\mathrm{III}}$ \\ Luiz Alexandre Peternelli' ${ }^{\mathrm{IV}}$ João Carlos Bespalhok Filho ${ }^{\text {II }}$ Edelclaiton Daros $^{\text {II }}$
}

RESUMO

Para a avaliação de experimentos com canade-açúcar, tem-se recomendado a colheita total de parcelas, algo que, devido aos seus custos, tem se mostrado inexequivel. Alternativamente, pode-se estimar a produção com base na tomada de amostras que representem as parcelas. $O$ objetivo deste trabalho foi estudar a suficiencia amostral em cana-de-açúcar para a estimação da massa dos colmos de parcelas experimentais. Reamostragens com reposição subsidiaram o uso dos modelos da curva potencial, linear e quadrático segmentados com resposta platô e do modelo do coeficiente de variação relativo. Também foram realizados estudos simulados de análise de variância. Os resultados indicaram que amostras de 20 colmos coletados aleatoriamente na parcela experimental permitem estimar adequadamente a variável massa do colmo. Com este valor, serão obtidas avaliações confiáveis da produtividade em colmos.

Palavras-chave: tamanho da amostra, suficiência amostral, melhoramento da cana-de-açúcar, Saccharum spp.

\section{ABSTRACT}

During the evaluation of the experimental phase, which corresponds to competition experiments and indication of cultivars, is recommended total harvest of plots, which, owing to their costs, can become impracticable. Optionally, one can estimate the production based on taking samples representing plots. The aim of this study was to research the sample sufficiency in sugarcane for estimating the mass of stalks of experimental plots. One used the maximum curvature of the power model, the linear and quadratic segmented models with response plateau and the relative $C V$ model. Studies based on simulated analysis of variance were done also. The application of these models and the ANOVA was supported by bootstrap resamplings. The results indicated that a sample made by 20 stalks harvested in plot can be used for estimating properly the variable mass of the stalk. This value may be used for estimating the sugarcane yield per hectare.

Key words: sample size, sample sufficiency, sugarcane breeding. Saccharum spp.

\section{INTRODUÇÃO}

A cana-de-açúcar (Saccharum spp.) encontra-se entre as mais importantes plantas cultivadas no mundo, estando entre os quatro mais importantes vegetais fornecedores de calorias à alimentação humana (ROSS-IBARRA et al., 2007). No Brasil, esta cultura tem ocupado mais de oito milhões e meio de hectares (CONAB, 2012), o que contribui para que o país seja o líder global na produção e exportação de produtos da cadeia sucroenergética (GIERSDORF, 2013).

Devido ao ciclo produtivo e fenológico da cultura, as diferentes fases do melhoramento da cultura sobrepõem-se no tempo, gerando-se altíssimas demandas por mão-de-obra, insumos e áreas experimentais. De fato, o melhoramento da cana-de-açúcar contempla pelo menos as seguintes fases experimentais: $\mathrm{T} 1$, correspondente ao cultivo e à avaliação dos seedlings obtidos por hibridação; fases intermediárias de avaliação e multiplicação vegetativa dos clones selecionados em $\mathrm{T} 1$, em número variável; e $\mathrm{FE}$, fase experimental, relativa

\footnotetext{
IPós-graduação em Agronomia, Produção Vegetal, Setor de Ciências Agrárias (SCA), Universidade Federal do Paraná (UFPR), 80060-000, Curitiba, PR, Brasil. E-mail: jga@ufpr.br. Autor para correspondência.

IDepartamento de Fitotecnia e Fitossanitarismo, SCA, UFPR, Curitiba, PR, Brasil.

IIIDepartamento de Estatística, Setor de Ciências Exatas, UFPR, Curitiba, PR, Brasil.

${ }^{\text {IV }}$ Departamento de Estatística, Universidade Federal de Viçosa, (UFV), Viçosa, MG, Brasil.
} 
à competição entre os clones mais promissores. Portanto, torna-se necessário que se otimize as operações envolvidas na condução das fases, especialmente aquelas relacionadas à fase $\mathrm{FE}$, a qual deve ser conduzida simultaneamente em diversos ambientes. A colheita total das parcelas das centenas de ensaios em FE, ainda que desejável, tem se tornado uma tarefa cada vez mais complicada e quase antieconômica de se realizar. Como opção, podem ser efetuadas estimativas confiáveis de produção a partir de técnicas de amostragem que indiquem o tamanho de amostra suficiente à estimação das variáveis que compõem o rendimento agrícola e industrial das culturas (suficiência amostral).

Qualquer amostra compõe-se de um determinado número de unidades básicas de amostragem, sendo evidente que, com o aumento do número de unidades básicas amostradas, a precisão e a confiabilidade das estimativas calculadas também serão incrementadas (SILVA et al., 1993). Porém, a partir de um determinado tamanho de amostra, incrementos no número de unidades básicas não resultam em proporcionais aumentos na precisão das estimativas, havendo pouca vantagem em se tomar amostras maiores (SILVA et al., 1993). Portanto, a fim de se validar uma recomendação de suficiência amostral, deve-se determinar o ponto crítico de desaceleração das medidas de variação entre os diferentes tamanhos de amostra possíveis. Isso tem sido feito mediante o emprego de metodologias capazes de descrever a variação frente ao incremento no tamanho da amostra.

Em substituição à colheita total de parcelas experimentais de cana-de-açúcar, LANDELL et al. (1999) avaliaram alguns métodos de amostragem para a estimação do índice de produtividade em colmos da cultura da cana-de-açúcar $(\mathrm{TCH})$ em áreas sob colheita mecanizada, enquanto que GHELLER et al. (1999) recomendaram a adoção de uma metodologia de amostragem para a avaliação indireta da produtividade, cujo enfoque concentrava-se mais nos procedimentos de amostragem em si do que no dimensionamento exato da amostra a ser coletada a campo. Nesse sentido, o objetivo deste trabalho foi estudar a suficiência amostral para a estimativa da variável massa do colmo (MC), que é o principal componente estimável de $\mathrm{TCH}$, estabelecendo uma recomendação segura do tamanho da amostra a ser coletada nas parcelas dos ensaios de competição de clones de cana-de-açúcar.

\section{MATERIAL E MÉTODOS}

O experimento foi conduzido no âmbito do Programa de Melhoramento Genético da Cana- de-açúcar da Universidade Federal do Paraná, na subestação da cidade de Colorado, região centro-norte do estado do Paraná, cuja área situa-se às coordenadas $22^{\circ} 45^{\prime} 17,45^{\prime}$ 'S e $51^{\circ} 56^{\prime} 38,60$ " W. O clima regional classifica-se como Cfa (subtropical úmido), com verões tépidos e chuvosos e invernos amenos. A área experimental encontra-se sobre Latossolo Vermelho distrófico de textura arenosa, num relevo "plano a suave ondulado", a uma altitude de 400 metros.

O campo experimental, correspondente à FE, foi instalado no dia 18 de junho de 2010. O experimento consistia de 30 clones promissores oriundos de cruzamentos realizados na Estação de Florescimento e Cruzamento da Serra do Ouro, localizada no município de Murici, estado de Alagoas, no ano 2000 (série RB00). Destes clones, foram selecionados três para a elaboração do presente estudo. Adicionalmente, avaliou-se a cultivar padrão 'RB867515'. O delineamento utilizado foi o de blocos completos casualizados, com três repetições, tendo sido colhidas doze parcelas correspondentes aos três clones ('RB005151', 'RB006655', 'RB006638') e à cultivar 'RB867515'. As parcelas foram compostas de quatro linhas de 8,0 metros de comprimento, com espaçamento interlinear de 1,4 metros, resultando em uma área de $44,8 \mathrm{~m}^{2}$. A densidade média de plantio foi de 18 gemas por metro. Este tamanho de parcela é compatível com as dimensões aceitáveis para a avaliação da sua massa, de acordo com PONTES et al. (2008).

A colheita das parcelas, cujas avaliações se deram em primeira cana soca, ocorreu na semana compreendida pelos dias 09 de julho a 13 de julho de 2012, tendo sido realizada da seguinte forma: (1) reconhecimento das parcelas e contagem (com os colmos eretos) do número de colmos industrializáveis de cada parcela; (2) contagem e colheita de todos os colmos industrializáveis (considerados, individualmente, como sendo a unidade básica de amostragem) e (3) descarte dos "ponteiros" (corte no ponto de quebra) e pesagem individual de cada colmo colhido, com balança de campo, cuja precisão nominal era de 10 gramas.

Para a determinação da suficiência amostral, utilizaram-se os seguintes modelos matemáticos: (1) Modelo da Curva Potencial - CP; (2) Modelo Linear Segmentado com Platô - LSP; (3) Modelo Quadrático Segmentado com Platô - QRP; e (4) Modelo do Coeficiente de Variação Relativo - CVR. Os modelos linear segmentado com resposta platô (LSP) e quadrático segmentado com resposta platô (QRP) foram ajustados aos conjuntos de dados obtidos de acordo com a sua descrição por FERREIRA (2011), a 
saber: $C V_{i}=b_{0}+b_{1} n_{i}, n_{i} \leq n_{0}$ e $C V_{i}=b_{0}+b_{1} n_{0}, n_{i}>n_{0}$, em que $b_{0}$ e $b_{1}$ são os parâmetros do segmento linear préplatô, $n_{i}$ é o i-ésimo tamanho amostral, $n_{0}$ é o valor da abscissa no ponto de união entre o segmento linear pré-platô e o segmento platô, representando o tamanho da amostral ideal (LSP); e $C V_{i}=c_{0}+c_{1} n_{i}+c_{2} n_{i}^{2}, n_{i} \leq n_{0}$ e $C V_{i}=c_{0}-\left(c_{1}^{2} / 4 c^{2}\right), n_{i}>n_{0}$, em que $c_{0}, c_{1}$ e $c_{2}$ são os parâmetros do segmento quadrático pré-platô, $n_{i}$ é o i-ésimo tamanho de amostra, $n_{0}$ é o valor da abscissa no ponto de união entre o segmento quadrático préplatô e o segmento platô, representando o tamanho ideal de amostra (QRP). No caso do modelo do $C V$ relativo (CARNEIRO et al., 2011), a curva foi ajustada a partir do seguinte modelo teórico:

$C V_{i}=d_{0}+\left(d_{1}-d_{0}\right) /\left(1+((1-q) / q)\left(\left(n_{i}-1\right) /\left(d_{2}-1\right)\right)\right)$ no qual $C V_{i}$ é o valor do $C V$ para uma amostra de tamanho $n_{i}, d_{0}$ é o valor do $C V$ para um tamanho amostral que tenda ao infinito, $d_{1}$ é o valor do $C V$ de uma amostra unitária, $d_{2}$ representa o tamanho amostral ideal para um valor de $q$ pré-determinado pelo pesquisador, relativo à amplitude máxima permitida para o valores da estatística $C V$. O diferencial deste modelo reside na possibilidade da sua resposta ser modulada pelo pesquisador, a partir da pré-determinação de um valor percentual $q$, o qual varia de 0 a 1 . Desse modo, adotando-se $q$ igual 0,15 , admite-se aceitar como ideal um tamanho de amostra cujo $C V$ seja $15 \%$ maior do que o mínimo, que corresponde a um valor de $q$ igual a zero, para o qual a amostra tenderia ao infinito.

Ao contrário dos demais modelos, cujos ajustes proporcionam diretamente as respostas relativas aos tamanhos ideais de amostra, no caso do modelo $\mathrm{CP}$, há que se calcular o ponto de máxima curvatura da sua função, a fim de se obter a resposta esperada. Sendo assim, os pontos de máxima curvatura deste modelo, cujos valores de abscissa correspondem ao tamanho de amostra ideal, foram determinados pelas seguintes proposições teóricas: (1) método da máxima curvatura modificado, ora abreviado como MCM I (MEIER \& LESSMAN, 1971), usando-se a expressão:

$x_{M C M I}=\left[a_{0}^{2}\left(a_{1}^{2}\left(2 a_{1}+1\right)\right) /\left(a_{1}+2\right)\right]^{\left[1 /\left(2 a_{1}+2\right)\right]}$ em que $x_{M C M I}$ representa o ponto de máxima curvatura da função, e $a_{0}$ e $a_{1}$ são os parâmetros da curva potencial, estimados por regressão não linear; (2) método da máxima curvatura modificado de BARROS \& TAVARES (1995), denominado MCM II. Este método se baseia na diferença entre a função da curva potencial ajustada e uma função linear, cuja reta passe pelos pontos correspondentes ao menor e ao maior tamanho de amostra comparados, sendo estes dois pontos as intersecções entre ambas as funções.
Dessa maneira, a fórmula usada no cálculo para a determinação do ponto de máxima curvatura foi: $x_{M C M I I}=\left(-a_{3} / a_{1} a_{0}\right)^{1 /\left(-a_{1}-1\right)}$, em que $x_{M C M I I}$ representa o ponto de máxima curvatura da função, $b_{2}$ corresponde ao coeficiente angular da reta supramencionada, e $b_{0}$ e $b_{1}$ são os parâmetros da curva potencial, estimados por regressão não linear. O modelo de curva potencial utilizado foi $C V_{i}=a_{0} / n_{i}^{a_{l}}$ (LOGAN, 2010), em que $n$ representa o i-ésimo tamanho de amostra.

Os ajustes dos modelos supramencionados foram subsidiados pela obtenção de dados através de reamostragens bootstrap (OYEYEMI, 2008). Foram executadas, para cada tamanho de amostra proposto, 1000 reamostragens com reposição, o que gerou 1000 reamostras distintas para cada tamanho amostral e para cada variável considerada. Estes procedimentos de amostragem aleatória foram realizados para cada uma das parcelas experimentais. Os tamanhos de amostra comparados (23, no total) foram os seguintes: $1,2,3,5,10,15,20,25,30,35,40,45,50,60,70$, $80,90,100,125,150,200,250$ e 507 colmos. O valor extremo, 507, corresponde ao número de colmos da parcela que apresentou o maior número de colmos.

A aplicabilidade da suficiência amostral obtida para a variável $M C$ foi testada pela competição de clones, a fim de se avaliar a sua efetividade na estimação do índice de produtividade em colmos por hectare $(T C H)$, calculado a partir dos dados biométricos de $M C$ (média, no caso de dados amostrais, ou massa total de parcelas) e de número de colmos por metro $(N C / M)$ através da seguinte proposição: $T C H=\overline{M C} \times N C / M^{2} \times 10^{-2}$.

Para a avaliação da performance do tamanho de amostra indicado pelo estudo da variável $M C$ na estimação do índice $T C H$, foram executadas 1000 análises de variância (ANOVA) simuladas para cada um dos 23 tamanhos amostrais anteriormente testados. Como a análise executada com os dados obtidos da colheita total das parcelas dos quatro clones efetivamente colhidos a campo não havia detectado diferenças estatísticas entre tais clones, incluiu-se um clone testador aos demais, cujos dados virtuais foram simulados pelo Programa R. Tanto as médias dos blocos quanto a média geral deste clone testador foram projetadas para serem semelhantes à média nacional de produtividade em colmos. Além disso, a média deste testador diferiu dos demais clones para um nível de significância $(\alpha)$ igual a 0,05 . Dos resultados daquelas 23000 análises e da análise executada com os dados obtidos da colheita total das parcelas ("ANOVA real"), incluindo-se os dados simulados para o clone testador, foram extraídos os seguintes itens de comparação: (i) os p-valores 
dos testes $\mathrm{F}$, a fim de se avaliar o poder do teste na detecção da diferença apontada pela "ANOVA real"; (ii) resultados das estatísticas $F$, para o enquadramento de cada simulação em uma determinada classe de precisão, conforme os seus respectivos valores de acurácia seletiva; e (iii) ranqueamento dos clones pelas suas médias de produtividade. Esses dados foram analisados e avaliados da seguinte forma: (i) análise dos p-valores obtidos pelos testes $\mathrm{F}$, para verificar-se, proporcionalmente, o número de vezes em que resultados dos p-valores foram significantes $(\alpha \leq 0,05)$, conforme o esperado; (ii) verificação do percentual de simulações que geraram análises de alta precisão, ou seja, cujos valores de acurácia seletiva foram maiores do que $70 \%$, correspondentes a valores de $\mathrm{F}$ maiores do que 2,0 (RESENDE \& DUARTE, 2007); e (iii) comparação entre os rankings obtidos pela correlação de postos de Spearman.

Para a implementação de todos os procedimentos estatísticos utilizados ao longo deste trabalho, lançou-se mão do aplicativo R de computação estatística (R DEVELOPMENT CORE TEAM, 2012).

\section{RESULTADOS E DISCUSSÃO}

Os gráficos gerados pelos valores de CV obtidos das 1000 reamostragens (para cara tamanho amostral e em cada parcela) evidenciam o comportamento inversamente proporcional dos $\mathrm{CV}$ com relação ao tamanho amostral (Figuras 1a, 1b, 1c, 1d). A análise prévia destes gráficos demonstra a existência de um momento crítico de desaceleração (estabilização) dos CV, a partir do qual haveria pouca vantagem em se aumentar a quantidade de unidades básicas amostradas (SILVA et al., 1993), sob pena de se aumentar excessivamente o tempo e os custos de amostragem para obter-se ganhos pouco expressivos na precisão experimental. Os elevados valores de $\mathrm{R}^{2}$ obtidos pelo ajuste do modelo $\mathrm{CP}$ assinalam a qualidade da sua adequação aos dados (Tabela 1), fato igualmente observado por ARAÚJO et al (2009) e por PEQUENO et al. (2003), que obtiveram $\mathrm{R}^{2}$ da ordem de 0,9686 e de 0,9800, respectivamente. Ademais, de acordo com o AIC (critério de informação de Akaike corrigido), dentre os modelos ajustados, este foi o que melhor explicou o comportamento do CV da variável (Tabela 1). No entanto, pode-se afirmar que os demais modelos também se adequaram satisfatoriamente aos dados, especialmente os modelos CVR e QRP.

Aplicando-se o método MCM I à curva potencial gerada para cada parcela, obteve-se um tamanho médio de amostra de 7 colmos (Tabela 1). Já pela aplicação do método MCM II, o valor médio obtido foi expressivamente maior: 35 colmos. Tais resultados corroboram a hipótese de Chaves (1985), apud OLIVEIRA et al. (2011), para quem o método da MCM I subestimaria os valores de abscissa dos pontos de máxima curvatura, representando muito mais um tamanho mínimo da amostra do que o ideal. Portanto, apesar do método da MCM I ter apresentado resultados satisfatórios para ARAÚJO et al. (2009), considerando-se que, nos experimentos em FE, apenas clones de ótima performance (clones elites) são postos em competição, havendo, no geral, sutis diferenças a serem detectadas entre eles, seria prudente não se basear somente neste método para a determinação do tamanho ideal de amostra para a avaliação da variável MC. Nesse sentido, para o dimensionamento da amostra a ser utilizada na seleção de famílias da fase T1 do melhoramento da cultura da cana-de-açúcar, o MCM I foi criticado por LEITE (2007). A seu turno, o método da MCM II, desenvolvido por BARROS \& TAVARES (1995) e até o momento sem registros de aplicação formal nos estudos de suficiência amostral, propiciou respostas condizentes com o que se tem verificado empiricamente. $\mathrm{O}$ valor médio obtido através deste método aproxima-se tanto dos tamanhos práticos de amostra recomendados por GHELLER et al. (1999) aos programas brasileiros de melhoramento genético da cana-de-açúcar, quanto do número sugerido por Mariotti \& Lascano (1969), apud ARIZONO et al. (1998), para quem 30 colmos ofereceriam boas estimativas da massa total da parcela. No entanto, em face da totalidade dos resultados obtidos, é possível que este método tenha incorrido em algum nível de superestimação. Considerando-se as sugestões de GHELLER et al. (1999) e o que empiricamente tem sido verificado a campo, o modelo CVR também poderia ser bem avaliado. Diante dos demais resultados, existe a possibilidade de que os modelos QRP e LSP tenham subestimado sutilmente o tamanho de amostra ideal. Quanto ao modelo LSP, contudo, PARANAÍBA et al. (2009) chegaram a resultados satisfatórios, utilizando esta metodologia.

Havendo modelos e métodos que parecem subestimar o tamanho suficiente de amostra e outros que talvez o superestimem, uma recomendação prática (20 colmos) baseada num valor de compromisso (valor médio) entre os resultados obtidos por todas as metodologias (Tabela 1), seria algo bastante razoável, contemplando fatores como a qualidade dos ajustes dos modelos e possíveis subestimações ou superestimações existentes nos resultados. De fato, a existência de 

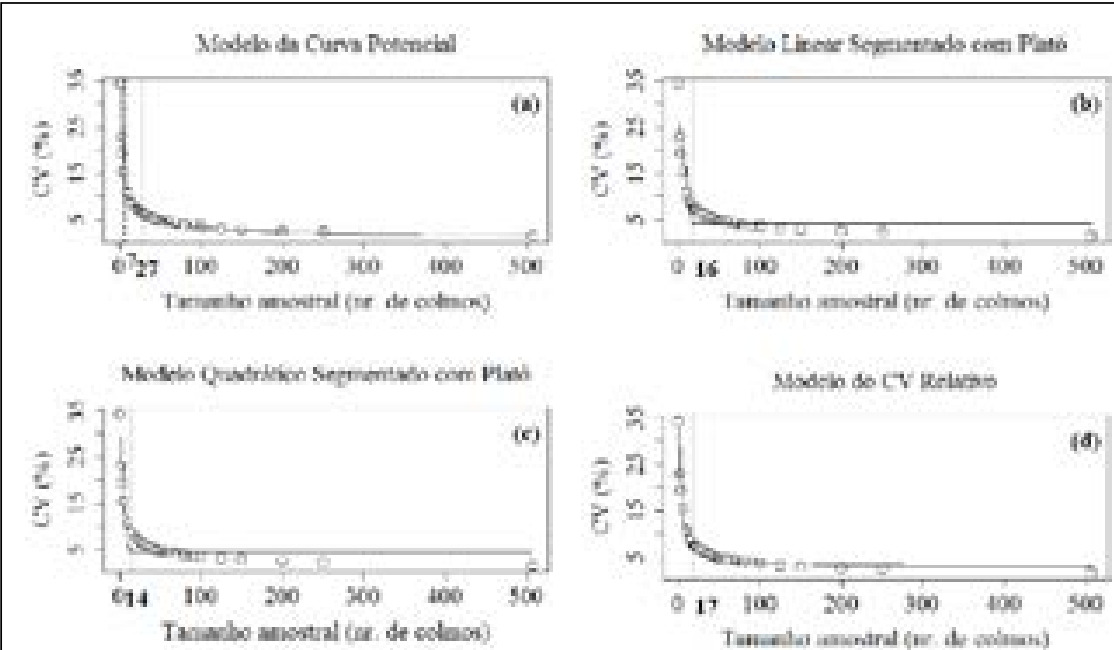

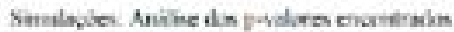
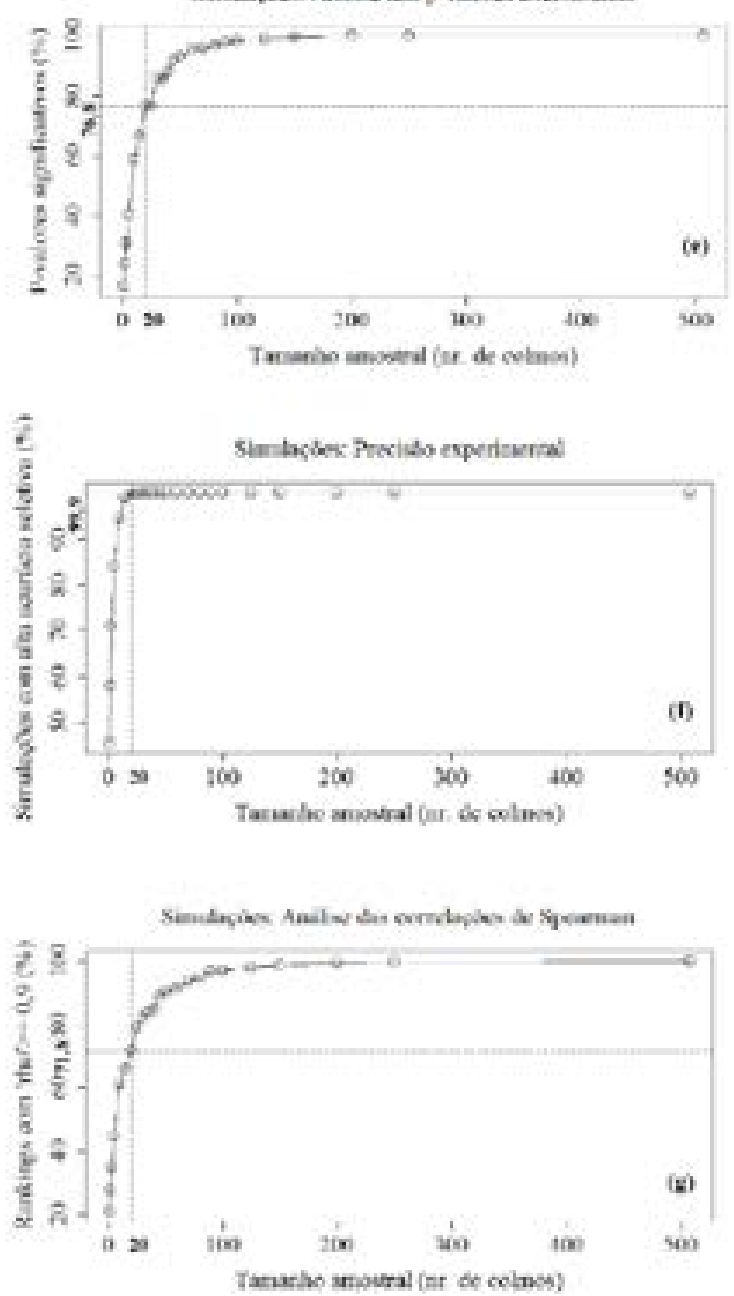

Figura 1 - Ajustes dos modelos CP (a), LSP (b), QRP (c) e CVR (d), para uma parcela (bloco um) da cultivar padrão. Simulações (\%) com p-valor significativo (e), de alta precisão (f), e proporções dos rankings cujos valores da correlação de Spearman foram maiores do que 0,9 (g). CP, modelo da curva potencial; LSP, modelo linear segmentado com platô; QRP, modelo quadrático segmentado com platô; e CVR, modelo do CV relativo. 
Tabela 1 - Tamanhos ideais de amostra ( $\mathrm{n}^{\mathrm{o}}$ de colmos), valores de $\mathrm{R}^{2}$ e AIC (critério de Informação de Akaike corrigido) obtidos pelos ajustes dos modelos da curva potencial (CP), linear segmentado com platô (LSP), quadrático segmentado com platô (QRP) e do CV relativo (CVR). Para o modelo CP, MCM I e MCM II referem-se aos dois métodos de cálculo da máxima curvatura utilizados neste trabalho. Todos os resultados foram arredondados para o maior valor inteiro subsequente.

\begin{tabular}{|c|c|c|c|c|c|}
\hline \multirow{2}{*}{ Clone / Bloco } & \multicolumn{5}{|c|}{ 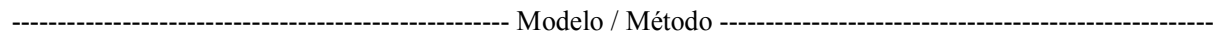 } \\
\hline & $\mathrm{CP} / \mathrm{MCM} \mathrm{I}$ & CP / MCM II & LSP & QRP & $\operatorname{CVR}(\mathrm{q}=0,15)$ \\
\hline RB867515/1 & 7 & 27 & 16 & 14 & 17 \\
\hline $\mathrm{R}^{2}$ & \multicolumn{2}{|c|}{0,9990} & 0,8805 & 0,9155 & 0,9891 \\
\hline AIC & \multicolumn{2}{|c|}{7,5701} & 120,9356 & 112,9665 & 65,8824 \\
\hline RB867515 / 2 & 6 & 34 & 16 & 14 & 17 \\
\hline $\mathrm{R}^{2}$ & \multicolumn{2}{|c|}{0,9995} & 0,8940 & 0,9252 & 0,9933 \\
\hline AIC & \multicolumn{2}{|c|}{$-16,0275$} & 111,9488 & 103,9472 & 48,5917 \\
\hline RB867515/3 & 7 & 34 & 16 & 18 & 20 \\
\hline $\mathrm{R}^{2}$ & \multicolumn{2}{|c|}{0,9996} & 0,8776 & 0,9146 & 0,9902 \\
\hline AIC & \multicolumn{2}{|c|}{$-17,5065$} & 115,8417 & 107,5569 & 57,8811 \\
\hline RB005151 / 1 & 6 & 38 & 16 & 14 & 17 \\
\hline $\mathrm{R}^{2}$ & \multicolumn{2}{|c|}{0,9995} & 0,8904 & 0,9253 & 0,9926 \\
\hline AIC & \multicolumn{2}{|c|}{$-10,3770$} & 117,8174 & 108,9971 & 55,7377 \\
\hline RB005151 / 2 & 6 & 39 & 16 & 14 & 18 \\
\hline $\mathrm{R}^{2}$ & \multicolumn{2}{|c|}{0,9993} & 0,8778 & 0,9132 & 0,9894 \\
\hline AIC & \multicolumn{2}{|c|}{$-10,7858$} & 112,0763 & 104,2175 & 55,8948 \\
\hline RB005151/3 & 5 & 42 & 16 & 14 & 19 \\
\hline $\mathrm{R}^{2}$ & \multicolumn{2}{|c|}{0,9988} & 0,8665 & 0,9207 & 0,9902 \\
\hline AIC & \multicolumn{2}{|c|}{4,5690} & 116,6343 & 104,6648 & 56,4895 \\
\hline RB006655 / 1 & 6 & 36 & 16 & 14 & 17 \\
\hline $\mathrm{R}^{2}$ & \multicolumn{2}{|c|}{0,9998} & 0,8858 & 0,9219 & 0,9921 \\
\hline AIC & \multicolumn{2}{|c|}{$-26,7848$} & 122,7890 & 114,0354 & 61,4042 \\
\hline RB006655 / 2 & 7 & 34 & 12 & 14 & 18 \\
\hline $\mathrm{R}^{2}$ & \multicolumn{2}{|c|}{0,9991} & 0,8775 & 0,9141 & 0,9891 \\
\hline AIC & \multicolumn{2}{|c|}{1,1512} & 116,2204 & 108,0451 & 60,5237 \\
\hline RB006655 / 3 & 7 & 31 & 16 & 14 & 17 \\
\hline $\mathrm{R}^{2}$ & \multicolumn{2}{|c|}{0,9993} & 0,8843 & 0,9196 & 0,9908 \\
\hline AIC & \multicolumn{2}{|c|}{$-15,3023$} & 106,4323 & 98,0665 & 48,2521 \\
\hline RB006638 / 1 & 6 & 33 & 16 & 15 & 19 \\
\hline $\mathrm{R}^{2}$ & \multicolumn{2}{|c|}{0,9996} & 0,8755 & 0,9167 & 0,9906 \\
\hline AIC & \multicolumn{2}{|c|}{0,9996} & 115,3958 & 106,1494 & 55,8872 \\
\hline RB006638 / 2 & 6 & 36 & 16 & 10 & 15 \\
\hline $\mathrm{R}^{2}$ & \multicolumn{2}{|c|}{0,9991} & 0,8833 & 0,9189 & 0,9901 \\
\hline AIC & \multicolumn{2}{|c|}{4,2426} & 120,2621 & 111,9028 & 63,5547 \\
\hline RB006638 / 3 & 6 & 35 & 16 & 13 & 17 \\
\hline $\mathrm{R}^{2}$ & \multicolumn{2}{|c|}{0,9991} & 0,8908 & 0,9183 & 0,9914 \\
\hline AIC & \multicolumn{2}{|c|}{7,7763} & 121,3941 & 114,7035 & 63,0390 \\
\hline Média & 7 & 35 & 16 & 14 & 18 \\
\hline Média geral & & & 18 & & \\
\hline Indicação prática & & & 20 & & \\
\hline
\end{tabular}

resultados intermediários, como aquele fornecido pelo modelo CVR corrobora esta opção (18 colmos).

Dos resultados das 23000 ANOVA simuladas, nota-se que amostras de tamanho igual a 20 colmos, quando usadas para o cálculo do índice de produtividade em colmos, geraram análises com razoável poder de detecção de uma determinada diferença estatística entre os índices TCH dos clones em competição. De fato, efetuando-se simulações de ANOVA com este tamanho amostral, 76,8\% dos p-valores (Figura 1e) vinculados aos testes $F$ das simulações detectaram a diferença existente ( $p$-valor 
igual a 0,011) entre o clone testador e os demais clones, ou seja, foram iguais ou menores a 0,050 . Não foram localizados resultados, em cana-de-açúcar, aos quais estes pudessem ser diretamente comparados. No entanto, na avaliação de tamanhos de amostra e métodos de amostragem em milho, SILVA et al. (1998) encontraram diferenças nos resultados da interpretação da estatística $\mathrm{F}$ (dos p-valores, por conseguinte) com o aumento do tamanho da amostra, para os caracteres de comprimento e peso da espiga. Em termos de qualidade experimental, praticamente todas as simulações efetuadas $(99,9 \%)$ corresponderam a análises de "alta precisão" (Figura 1f), sendo assim classificadas por apresentarem valores de $\mathrm{F}$ superiores a 2,0, correspondentes a valores de acurácia seletiva maiores do que 0,7 . No melhoramento vegetal, experimentos cujos níveis de acurácia seletiva sejam maiores do que $70 \%$ poderiam ser aproveitados para procedimentos de seleção (RESENDE \& DUARTE, 2007). Comparando-se os rankings obtidos das 1000 ANOVA simuladas com o ranking oriundo da "ANOVA real", através da correlação de postos de Spearman $(\rho)$, mais de $70 \%$ dos rankings gerados (Figura 1g), no caso das amostras compostas por 20 colmos, forneceram resultados com apenas uma alteração de ordem (valores de $\rho$ iguais ou maiores do que 0,9 ).

O tamanho de amostra indicado por este trabalho como sendo suficiente para o cálculo da estimativa de $\mathrm{MC}$ e aplicável à composição do índice de produtividade TCH pode ser, pela sua dimensão, utilizado nas rotinas de amostragem dos programas de melhoramento genético de cana-de-açúcar, otimizando-se a utilização dos recursos destinados às avaliações e abreviando-se o tempo necessário à obtenção dos dados advindos do campo.

\section{CONCLUSÃO}

A massa média de vinte colmos industrializáveis, aleatoriamente coletados, é suficiente para a estimação da massa de parcelas experimentais de cana-de-açúcar. Este tamanho de amostra demonstrou ser adequado ao cálculo do índice de produtividade da cultura da cana-de-açúcar (TCH). Análises de variância executadas com dados obtidos de amostras com tais dimensões possuem alta qualidade experimental, constituindo-se numa opção aceitável às análises efetuadas mediante a colheita total de parcelas.

\section{AGRADECIMENTOS}

À Rede Interuniversitária para o Desenvolvimento do Setor Sucroenergético (RIDESA) por ter oportunizado a realização deste trabalho e à Coordenação de Aperfeiçoamento de Pessoal de Nível Superior (CAPES) pela concessão de bolsa de estudos.

\section{REFERÊNCIAS}

ARIZONO, H. et al. Alternativas para avaliação de produção de cana-de-açúcar. Stab, Piracicaba, v.16, n.5, p.20, 1998.

ARAÚJO, E.G. et al. Métodos de amostragem e tamanho de amostra para avaliar o estado de maturação da uva niágara rosada. Revista Brasileira de Biometria, São Paulo, v.27, n.4, p.501-518, 2009.

BARROS, I.; TAVARES, M. Estimativa do tamanho ótimo de parcelas experimentais através de cálculos algébricos. Bragantia, Campinas, v.54, n.1, p.209-215, 1995. Disponível em: <http://dx.doi. org/10.1590/S0006-87051995000100024>. Acesso em 15 ago. 2012.

CARNEIRO, F.F. et al. Minimum number of common bean plants per plot to assess field resistance to white mold. Crop Breeding and Applied Biotechnology, Viçosa, v.11, p.358-364, 2011. Disponível em: <http://www.sbmp.org.br/cbab/siscbab/uploads/ bd534dc5-2c80-70b2.pdf>. Acesso em 05 ago. 2012.

CONAB (COMPANHIA NACIONAL DE ABASTECIMENTO), Acompanhamento de safra brasileira: cana-de-açúcar, terceiro levantamento, dezembro/2012 - Companhia Nacional de Abastecimento. Brasília, 2012. 16p.

FERREIRA, D.F. Recursos computacionais utilizando R. Lavras: Universidade Federal de Lavras, 2011. 228p.

GHELLER, A.C.A. et al. Manual de método alternativo para medição da produção de cana-de-açúcar. Araras: UFSCar CCA - DBV, 1999. 7p.

GIERSDORF, J. Politics and economics of ethanol and biodiesel production and consumption in Brazil. Deutsches Biomasseforschungszentrum, 2013. Leipzig: DBFZ Report n.15.

LANDELL, M.G.A. et al. Validação de métodos de amostragem para a estimativa da produção de cana-de-açúcar, em áreas de colheita mecanizada. Stab, Piracicaba, v.18, n.2, p.48-51, 1999.

LEITE, M.S.O. Tamanho da amostra para seleção de famílias de cana-de-açúcar. 2007. 51f. Dissertação (Mestrado em Genética e Melhoramento) - Universidade Federal de Viçosa, Viçosa, MG.

LOGAN, M. Biostatistical design and analysis using $\mathbf{R}-\mathbf{a}$ practical guide. Oxford, UK: Wiley-Blackwell, 2010. 546p.

MEIER, V.D.; LESSMAN, K.J. Estimation of optimum field plot shape and size testing yield in Crambe abyssinica HORDNT. Crop Science, Madison, v.11, p.648-650, 1971. Disponível em: $<$ http://dx.doi.org/10.2135/cropsci1971.0011183X00110005001 $3 \mathrm{x}>$. Acesso em: 15 ago. 2012.

OLIVEIRA, G.M.V. et al. Tamanho e forma de parcelas experimentais para Eremanthus erythropappus. Cerne, Lavras, v.17, n.3, p.327-338, 2011.

OYEYEMI, G.M. Comparison of bootstrap and jackknife methods of resampling in estimating population parameters. Global 
Journal of Pure and Applied Sciences, v.14, n.2, p.217-220, 2008. Disponível em: <http://dx.doi.org/10.4314/gjpas.v14i2.16802>. Acesso em: 10 set. 2012

PARANAÍBA, P.F. et al. Tamanho ótimo de parcelas experimentais: proposição de métodos de estimação. Revista Brasileira de Biometria, São Paulo, v.27, n.2, p.255-268, 2009.

PEQUENO, S.A. et al. Determinação do número de marcadores RAPD para estudos da diversidade genética em soja utilizando o método bootstrap. Bioscience Journal, Uberlândia, v.19, n.2, p.45-48, 2003.

PONTES, M.S. et al. Estimativa do tamanho de parcela em cana-de-açúcar pelo método da máxima curvatura modificado. In: CONGRESSO NACIONAL DA STAB - SOCIEDADE DOS TÉCNICOS AÇUCAREIROS E ALCOOLEIROS DO BRASIL, 2008, Maceió, AL. Anais... Maceió: STAB, 2008; p.636-640.

R CORE DEVELOPMENT TEAM. R: a language and environment for statistical computing. Vienna, Austria: R Foundation for Statistical Computing, 2012. Disponível em: $<$ http://www.R-project.org/>. Acesso em: 05 mar. 2012.
RESENDE, M.D.V.; DUARTE, J.B. Precisão e controle de qualidade em experimentos de avaliação de cultivares. Pesquisa Agropecuária Tropical, Goiânia, v.37, n.3, p.182-194, 2007. Diposnível em <http://www.revistas.ufg.br/index.php/pat/article/ view/1867/1773>. Acesso em: 15 jun. 2013.

ROSS-IBARRA, J. et al. Plant domestication, a unique opportunity to identify the genetic basis of adaptation. Proceedings of the National Academy of Sciences, USA, v.104, suppl.1, p:8641-8648, 2007. Disponível em: <http://dx.doi.org/10.1073/ pnas.0700643104>. Acesso em 10 jun. 2013.

SILVA, J. et al. Amostragem e tamanho da amostra na estimação de caracteres da espiga do milho. Pesquisa Agropecuária Brasileira, Brasília, v.33, n.12, p.1983-1988, 1998. Disponível em: <http:// seer.sct.embrapa.br/index.php/pab/article/view/5030/7179>. Acesso em 25 ago. 2012.

SILVA, P.S.L. et al. Métodos de amostragem e tamanho da amostra para alguns caracteres do milho. Ciência Agronômica, Fortaleza, v.24, n.1/2, p.5-10, 1993. Disponível em: <http://www.ccarevista. ufc.br/site/down.php?arq=01rca24.pdf>. Acesso em: 02 set. 2012. 